\title{
Polysèmes
}

Revue d'études intertextuelles et intermédiales

17 | 2017

L'art intempestif-La démesure du temps

\section{Francis Bacon, un moderne intempestif}

\section{Annie Mavrakis}

\section{(2) OpenEdition}

\section{Journals}

Édition électronique

URL : http://journals.openedition.org/polysemes/1881

DOI : 10.4000/polysemes. 1881

ISSN : 2496-4212

Éditeur

SAIT

\section{Référence électronique}

Annie Mavrakis, « Francis Bacon, un moderne intempestif », Polysèmes [En ligne], 17 | 2017, mis en ligne le 30 avril 2017, consulté le 20 avril 2019. URL : http://journals.openedition.org/polysemes/1881 ; DOI : 10.4000/polysemes. 1881

Ce document a été généré automatiquement le 20 avril 2019

Polysèmes 


\title{
Francis Bacon, un moderne intempestif
}

\author{
Annie Mavrakis
}

1 Parler d'art intempestif suppose de prendre en compte ce qu'est - ce que serait - pour nous aujourd'hui un art non intempestif: inscrit dans l'horizon d'attente du présent, donc entrant dans les catégories de ce que l'on a pris l'habitude d'appeler globalement $l a$ modernité. Question évidemment piégée puisque notre vision de l'art comme essentiellement résistant, novateur, en rupture sacrifie à un stéréotype historiquement daté tendant à faire oublier que, dans le passé, le plus original des artistes s'inscrivait dans une tradition. Si bien que nous nous trouvons devant une aporie de même nature que cette «tradition du nouveau » dont a parlé Harold Rosenberg ${ }^{1}$, l'injonction réputée intempestive de rompre (avec quoi désormais ?) s'avérant rien moins qu'intempestive.

2 J'ai fait appel à Francis Bacon d'abord parce que ce peintre, de façon tout à fait consciente, est, au premier sens du terme, intempestif; par son refus maintes fois formulé d'une histoire de l'art orientée, "progressiste », il se place résolument, de façon quasi militante, à contresens plutôt qu'en marge des pratiques hégémoniques de son époque et de ses prescriptions (non figuration, non narration, non forme, etc.). Mais quand Bacon regrette que l'artiste d'aujourd'hui soit "outside a tradition" (Sylvester 2008, 43), ce n'est pas, comme on pourrait le croire, par nostalgie : son œuvre est moderne en ce qu'elle n'est intelligible qu'en référence à du déjà-là : non le « cliché » encombrant la toile dont parle Deleuze ${ }^{2}$ mais un classicisme qui, loin de n'être qu'un dépôt fossilisé à jeter au feu pour "avancer", ressurgit de façon imprévue, colorant diversement les productions nouvelles, "modernes» chaque fois différemment. Cette modernité-là, qui est aussi celle de Yeats et d'Eliot, implique à la fois un rapport au passé et d'avoir compris que «le passé n'est pas passé » (Faulkner), qu'il fait partie de notre présent ${ }^{3}$.

3 Cette idée était présente dans la fameuse définition baudelairienne de la modernité, souvent citée, presque toujours tronquée : «la modernité, c'est le transitoire, le fugitif, le contingent, la moitié de l'art, dont l'autre moitié est l'éternel et l'immuable », écrivait le 
poète dans Le Peintre de la vie moderne. Deux moitiés, dont la première ne périme ni n'annule la seconde mais en rebrasse, en transforme les éléments au sein même de l'œuvre, apportant à des questions séculaires (composition, perspective, rapport aux modèles ou à la nature, primat du dessin ou du coloris) des réponses parfois déroutantes et pourtant aptes à modifier de façon irréversible la vision du public.

Or, à l'époque de Bacon, c'est-à-dire l'après-guerre, où l'antonyme de « moderne » n'est plus « classique " mais « dépassé », « rétrograde », voire « réactionnaire ", cette " double appartenance » de l'art ${ }^{4}$ semble impossible. Comme le déplore Leiris dans son Journal (14 juin 1969), on a pris l'habitude d'évaluer les artistes « selon la place plus ou moins avancée qu'ils occupent dans l'évolution de l'art » (Leiris 1992, 631). La notion même de modernité s'étant - à force de généralité - vidée de son sens, seules subsistent deux options: soit être "contemporain", suivre perpétuellement le courant, soit, en vrai moderne, «jeter sur le tapis cette carte majeure : nos rapports avec le réel, notre façon d'appréhender le monde» (Leiris 1980, 16), c'est-à-dire renouer un fil rompu depuis longtemps. S'appuyant sur ceux qu'il a désignés comme «intempestifs »: «Giacometti, Balthus, Bacon » et même Picasso dont certains tableaux « presque naturalistes » relèvent d'une "peinture qui se veut peinture ", n'a pas "divorcé avec la tradition » et use de moyens «classiques » pour « exprimer » quelque chose (Leiris 1980, 24), Leiris, dans les différents textes qu'il a consacrés à la peinture, a défini les conditions de la refondation d'une autre modernité. On peut les résumer ainsi :

5 L'abandon du privilège absolu accordé à l'innovation formelle, que Leiris appelle le "préjugé quant aux moyens de dire » (Leiris 1992, 750, 27 mars 1982). Ce qui lui plaît dans les tableaux non cubistes de Picasso c'est l'idée que « tous les moyens sont bons y compris les plus ordinaires ». "Ce qui émeut alors », dit-il, «c'est le rejet à tout le moins apparent de toute recherche "esthétique" - les distances prises, en somme, avec les préoccupations d'ordre formel ».

6 L'affirmation du caractère transhistorique et donc anachronique de l'art, à contre-courant de l'amnésie qui enferme l'artiste dans son temps et voudrait « éliminer [des] œuvres tout élément "anachronique" » (Duchamp cité par Leiris [Leiris 1992, 370, 21 juin 1942]). Leiris rejoint en cela Genet ironisant sur ses contemporains qui prétendent travailler pour les "générations enfants ", dépourvues de mémoire 5 .

7 La remise à l'ordre du jour du réalisme, majoritairement discrédité comme reflet passif, décalque du réel. Leiris appelle réalisme une figuration subjective, créatrice, qui, dit-il, traduit la relation avec "le motif positif ou l'image mentale [...] d'où part l'auteur " (Leiris 1992, 751, 25 avril 1982). Figurer le monde, insiste Leiris, n'est pas « chosifie[r] ce que de nos jours on appellerait le référent » (Leiris 1992, 751, 25 avril 1982). La notion de réalisme est tout à fait centrale non seulement pour lui mais pour tous ceux qui, comme Bonnefoy, demandent à l'art de ne pas se détourner du monde. «Ce qu'il faut », dit Leiris, en cela baudelairien, «ce n'est pas une fuite devant le réel ou encore un tri qui n'en retiendrait que les beaux éléments (en somme les beautés toutes faites) mais une reprise qui, sans s'écarter de lui, donne au réel une beauté» (Leiris 1992, 732, 28 octobre 1980, je souligne).

8 Enfin la présence d' " un arrière-plan littéraire, à tout le moins imaginaire...» : « derrière sous-bois et forêt, il y a les contes de fées [...] et les romans de la Table Ronde; derrière le ruisseau, il y a le mystère des herbes aquatiques et du fond bourbeux et caillouteux, peutêtre aussi la légende d'Ophélie » (Leiris 1992, 657, 12 août 1972). 
Ces conditions, la dernière en particulier, après un siècle de préjugé anti-littéraire ${ }^{6}$, Bacon a tenté, à sa façon souvent douloureuse et contradictoire, de les remplir. Refusant d'appartenir à quelque école que ce soit ${ }^{7}$, ne manquant pas une occasion de tourner en dérision les pratiques contemporaines, il se distingue dès ses premières toiles d'autres « marginaux » figuratifs (notamment anglais) en ce que justement il ne se contente pas de l'être. Pris entre le souci extrême de la ressemblance et la défiance à l'égard des procédures mimétiques habituelles, il a conçu son travail comme une «négociation » serrée avec une « littéralité » devenue pour diverses raisons presque inaccessible. Pour Bacon, dont la peinture définit un espace à part marqué par un double rapport - réinventé - à la nature et à la fable, la question de la représentation, restitution de ce qu'il appelle le "fait », ne s'esquive pas. C'est même la seule question qui se pose aux artistes de son temps. Le « fait » est l'enveloppe extérieure des êtres ou des choses, cette " apparence » qui, insiste Bacon, « doit être rendue » (Sylvester 2008, 152). Mais le « fait » est aussi leur vérité, leur essence. Bacon a cherché toute sa vie des dispositifs capables de rendre réelles les images qui le hantent, il leur a (c'est son expression) «tendu des pièges »: « Les choses », a-t-il expliqué à Michael Peppiatt, «ne provoquent pas de choc si elles n'ont pas été mises en forme de façon mémorable. Sinon ce n'est que du sang éclaboussant un mur » (39).

Les motifs visuels tirés de certaines photographies (entre autres celles de Muybridge, photographe du corps humain en mouvement), les «sujets » empruntés à des tableaux religieux et mythologiques ou à des poèmes et des pièces de théâtre sont chargés d'affects et donc vecteurs d'émotion. L'ensemble constitue pour le peintre une «banque » d'images qui se «coagulent» avec des circonstances intimes pour donner naissance aux œuvres complexes que nous connaissons. Bacon n'a pas caché ces emprunts mais les étiquettes qui s'attachent à son art (« ni illustratif, ni narratif », « immédiat », etc.) ont découragé de chercher dans les sources avouées par le peintre autre chose qu'une vague atmosphère ("It's the whole atmosphere of it that affects one», explique Bacon à David Sylvester [Sylvester 2008, 152])8. Or, la présence des poètes (Eschyle, Yeats, Eliot mais aussi Shakespeare ou García-Lorca) est chez Bacon très insistante ; elle est fondamentale et lui dicte motifs et situations. De la même façon qu'il dit avoir «manipul[é] les corps de Muybridge en leur donnant la forme de corps qu'[il avait] connus ", c'est sur un théâtre imaginé par des poètes, peuplé de leurs personnages, qu'il a placé les icônes issues de ce laboratoire qu'était pour lui le portrait (celles de George Dyer, d'Isabel Rawsthorne, d'Henrietta Moraes, etc.), élevant la scène de genre et même l'anecdote vécue au rang de peinture d'histoire.

11 Il ne saurait être question ici d'étudier de façon exhaustive cet aspect essentiellement intempestif de l'œuvre de Bacon. Je me contenterai de quelques exemples - motifs et personnages - tirés d'une de ses principales sources : la poésie et le théâtre de T.S. Eliot, eux-mêmes nourris du souvenir d'Eschyle et de Sophocle. "Sweeney ", "l'homme au rasoir » est, sous les traits de son amant George Dyer (fig. 1), l'une des créatures que Bacon leur a empruntées. Dans plusieurs toiles, notamment Three Studies of a Male Back de 1970 (fig. 2), Dyer/Sweeney, un rasoir à la main, est assis dans un fauteuil de coiffeur. Popularisé depuis par le film de Tim Burton (Sweeney Todd), ce personnage légendaire de barbier assassin revient comme un leitmotiv dans plusieurs œuvres d'Eliot : Sweeney Erect (1920), Sweeney Among the Nightingales (1920), la troisième section de The Waste Land (1922) et Sweeney Agonistes, poème dramatique inachevé auquel un célèbre triptyque de 1967 doit son titre et qui s'ouvre sur une citation des Choéphores: "Vous ne les voyez pas, vous ", dit Oreste au Coryphée, «mais moi, je les vois. Elles me pourchassent, je ne puis plus 
rester ». Ce sont les paroles d'Oreste, lorsqu'après le matricide, il découvre les Érynies. La présence dans plusieurs tableaux de cette furie antique, que Bacon "profil[e] pour la plongée et le rapt» (selon la belle formule de Jacques Dupin) s'explique par une familiarité avec Eschyle et Eliot qui n'a rien de livresque mais procède d'une affinité profonde. Seated Figure (fig. 3), par exemple, se souvient du début de The Family Reunion, pièce d'Eliot dont le héros, nouvel Oreste revenu de voyage, voit apparaître des Erynies par la fenêtre du château familial.

Au début de Sweeney Erect, évoquant l'antique Ariane errant sur les rives de Naxos, le poète passe commande d'un tableau à caractère mythologique,

Paint me a cavernous waste shore

Cast in the unstilled Cyclades

Paint me the bold anfractuous rocks

Faced by the snarled and yelping seas. (Eliot 42)

mais deux strophes plus loin, le spectacle s'est réduit aux dimensions d'une chambre que l'on devine sordide, décor véritablement baconien où une silhouette semblable à celle d'un orang-outang ("Apeneck Sweeney»: on ne compte pas les silhouettes simiesques chez Bacon) s'extirpe de "draps fumants", tandis qu'un être au statut incertain ("the epileptic on the bed») s'agite sur le lit défait. Lecteur d'Eliot, Bacon a pris acte du rétrécissement auquel est soumis l'univers de l'artiste moderne, geste éminemment intempestif. L'antique tragédie se joue désormais dans une sorte de cage entre des êtres presque privés d'humanité, figures informes étalées sur des draps parfois sanglants ( $c f$. Three Studies for a Crucifixion, 1962, panneau central (fig. 4), ou Three Studies of Figure Lying on a Bed, 1972).

Dans Sweeney Among the Nightingales, Eliot a placé en épigraphe le cri d'agonie du fils d'Atrée assassiné à son retour de Troie : «Hélas un coup mortel a pénétré ma chair » ( Agamemnon). La dernière strophe fait entendre le hurlement du roi à l'extérieur, « among the nightingales $»^{9}$. Le barbier Sweeney, l'homme au rasoir, joue le rôle du tueur et s'identifie aux meurtriers antiques, d'autant plus aisément à Égisthe que ce dernier, à cause du festin d'Atrée, est associé au thème du cannibalisme.

Dans le deuxième fragment de Sweeney Agonistes, Sweeney propose à une jeune femme de l'emmener dans une "île cannibale $»^{10}$. Une atmosphère de terreur et de cauchemar s'installe aussitôt dans la pièce, avec l'allusion à un cadavre dissous dans une baignoire qui convoque de nouveau le souvenir d'Eschyle. Sans illustrer à proprement parler le poème d'Eliot, ou sa « source » antique, le panneau central du triptyque Sweeney Agonistes (fig. 5) montre dans une sorte de cabine de bateau les traces sanglantes d'un assassinat. Les bagages abandonnés suggèrent un retour de voyage: on repense à Agamemnon revenu à Mycènes pour s'y faire égorger après une désastreuse traversée.

coïncidence entre l'histoire de Sweeney et le premier volet de L'Orestie se développe dans les panneaux latéraux: des corps enchevêtrés y reposent sur une estrade insérée dans une sorte de cube, qui évoque fortement l'eccyclème antique. Clytemnestre dans Agamemnon et Oreste dans Les Choéphores y exposent à la vue du public les cadavres de leurs victimes enlacés par leurs soins dans une étreinte obscène. C'est aussi une moderne "scène de crime»: un personnage masculin au téléphone, un de ces témoins ou spectateurs («attendants ») chers à Bacon, est visible à droite dans le miroir.

Le souvenir d'Eschyle et d'Eliot est encore présent dans le motif de la porte, essentiel chez Bacon et dont le traitement est tout à fait caractéristique de la façon dont travaille ce peintre: à la fois abstraite et figurée avec précision, en perspective, sans oublier 
baguettes, poignées et interrupteurs modernes. Derrières ces portes familières, en effet, se profilent celles du palais des Atrides à Mycènes, «qui sent le meurtre et le sang répandu »; et les voilà chargées de toute l'angoisse venue du mythe. Dans Agamemnon, Cassandre, évoquant l'atroce festin offert à Thyeste par son frère, qualifie d'«abattoir humain au sol trempé de sang " le lieu où elle vient d'être amenée, ce qui explique le surgissement du moderne Sweeney dans l'univers d'Eliot d'abord puis dans celui de Bacon. Au moment où le vainqueur de Troie s'avance vers l'entrée, la prophétesse s'écrie : "Je salue dans ces portes les portes de l'Enfer".

Chez Bacon aussi, les portes matérialisent la séparation entre les vivants et les morts. On ne les franchit pas, et même on ne s'en approche pas sans risque. Le peintre inscrit ainsi dans l'espace même de ses tableaux le néant qui menace la figure. Dans le Triptych MayJune 1973 (fig. 6), les trois moments de l'agonie de George Dyer tels que Bacon les a reconstitués se découpent dans le cadre d'une porte impitoyablement béante.

Deux sinistres portes encore dans le Triptych inspired by Oresteia of Aeshylus (fig. 7) où Bacon reprend le motif de l'Érynie. Sur les panneaux latéraux, une porte entrouverte, comme bloquée dans cette position par la structure emboîtée à l'intérieur. À gauche, une coulée de sang. L'Érynie est là, attirée par l'odeur de mort. À droite, elle semble s'être emparée d'un corps qu'elle fait littéralement passer au travers de la porte : il est happé vers le centre du triptyque où il réapparait cassé en deux, la colonne vertébrale à nu exposée verticalement à la façon d'une carcasse dans un abattoir. Le tapis rouge est peut-être encore un souvenir des étoffes que la Clytemnestre d'Eschyle a fait disposer sous les pieds de son époux juste avant de l'assassiner.

Ici, l'Érynie a été la plus forte : il ne reste pas grand-chose du personnage une fois qu'elle lui a fait franchir la porte fatidique. Elle résiste pourtant comme semblent le montrer des tableaux de la même époque où la figure tente avec plus ou moins de succès d'échapper à son destin. Déjà le Triptych August 1972 représentait les menaçants rectangles noirs tout en ménageant une distance possible pour la figure. Dans EEdipe et le Sphinx (1983), l'Érynie semble frappée à mort cependant qu'(Edipe survivant panse son pied blessé. Une étude de 1986 pour un portrait de John Edwards (fig. 8) reprend le rectangle noir compact sur fond de mur gris verdâtre. C'est bien une porte avec interrupteur et baguette électrique. Le visage du modèle aux traits comme écrasés sous un bas, semble irrésistiblement aspiré par cette ouverture menaçante : son corps se désagrège, la matière grisâtre dont il est constitué se met à couler. Une jambe et un pied ont déjà disparu. Mais l'ombre rouge fait contrepoids et maintient tant bien que mal le personnage dans l'espace de la représentation.

Study for the Human Body de 1987 (fig. 9) retravaille à peu près les mêmes éléments en les simplifiant. Au sol, l'ombre se confond avec la jambe gauche et en quelque sorte l'aide à résister à l'anéantissement. Une flèche blanche fixe la silhouette, l'épingle presque comme pour l'empêcher d'aller plus loin et de s'engouffrer dans l'obscurité. On note que les formes sont nettement moins défaites que dans l'étude de 1986. Dans la jambe droite, très musclée, éclairée, se concentre visiblement la résistance de tout le corps. Un portrait de $1988^{11}$ le montre sereinement assis à distance de la porte, encore lesté par l'ombre de son corps et presque hors de danger.

21 Cette porte-trappe effrayante, tentatrice peut-être, est partout. L'un des motifs les plus étranges et impressionnants que Bacon ait utilisés, celui de la clé introduite dans la serrure, également présent dans de petites peintures faites à Dinard par Picasso à la fin des années 20 et que Bacon aimait beaucoup (fig. 10 et 11) ${ }^{12}$, lui est associé. Bacon avait 
parfaitement conscience de son origine littéraire: "Je travaille actuellement à un triptyque d'après les vers d'Eliot: “J'ai entendu la clé tourner dans la porte une fois et une fois seulement" " (citant de mémoire et en français, Bacon abrège le texte de The Waste land). Et à David Sylvester $(2008,150)$ à propos de Painting: «It very much came from that poem of Eliot's: "I have heard the key / Turn in the door once and turn once only..." You know. It comes from The Waste Land». À la fin de Sweeney Agonistes, apparait en effet le motif de la clé qui tourne dans la serrure ${ }^{13}$, annonçant l'arrivée à l'aube d'un visiteur terrifiant: the "hoo-ha» (la Mort? le bourreau? Il est question plus loin du " hangman », le Pendu du tarot ou celui qui pend $\left.{ }^{14}\right)$ : «And you wait for a knock and the turning of a lock / for you've got the hoo-ha's coming to you », effet renforcé par la transcription des neuf coups sur la porte: "KNOCK KNOCK KNOCK / KNOCK KNOCK KNOCK / KNOCK / KNOCK / KNOCK ». Les cris (« hoo hoo hoo») font écho au nom du bourreau comme dans une comptine enfantine qui s'élèverait sur un carnage.

Associé à la porte, le motif du «turning of the lock » suffit désormais à introduire dans l'œuvre de Bacon un climat d'angoisse. Bacon l'introduit en 1967 dans un triple portrait d'Isabel Rawsthorne (fig. 13) qui inscrit différents modes de représentation: l'image derrière la porte étant l'icône figée de celle qui, encore vivante, tente de la verrouiller mais trop tard comme le montre l'autre icône épinglée à l'intérieur de la pièce.

Le peintre a eu recours au motif de la clé à plusieurs reprises après le suicide de George Dyer, notamment dans le panneau central du triptyque à sa mémoire (fig. 14). On y voit la porte ouverte avec la clé fatale par une figure placée dans l'ombre où l'on reconnaît le profil de George Dyer. La cage d'escalier peu éclairée reproduit méticuleusement celle de l'Hôtel des Saints-Pères où eut lieu le drame. La pièce qui ouvre sur le palier, avec son ampoule, est en outre une quasi-citation du panneau central du Triptych May-June 1973. Une fois de plus, les panneaux latéraux offrent plusieurs types de représentation: à gauche, l'agonie reconstituée. À droite, l'icône du mort, presque stabilisée malgré la duplication en miroir. La bande bordeaux, couleur de sang séché, qui court sur toute la largeur du triptyque évoque de nouveau le piège de pourpre de Clytemnestre. Comme l'a bien vu Sylvester, l'œuvre est faite des « trois images superposées qui hantaient Bacon celle de Picasso, celle d'Eliot et l'événement lui-même » (Sylvester 1996, 27). Un moment de pure terreur a ainsi été «enregistré ». Tout en collant aux circonstances, Bacon a su donner un caractère grandiose à la commémoration.

On retrouve le motif du «turning of the lock» dans une peinture de 1978, d'abord intitulée "Eliot ». Comme dans le Triptych à la mémoire de George Dyer, il est associé à l'image dédoublée du mort : ombre accompagnant la silhouette à la jambe tendue mais aussi buste vu de profil et emboîté dans une espèce de cage, ce qui donne l'impression que, bien que placé juste derrière le mur, le personnage est aussi radicalement hors de portée qu'Eurydice perdue une seconde fois. Une flèche attire l'attention sur Dyer et, dans son prolongement, une autre flèche désigne un journal aux caractères brouillés qui annoncent peut-être déjà - mais aussi indéchiffrables qu'un oracle antique - la nouvelle du suicide

Jamais comme dans ces toiles, Bacon n'aura été conduit si près de la restitution «presque littérale » qu'il admirait chez Rembrandt, Vélasquez ou Van Gogh. Mais ses œuvres n'ont une telle puissance que parce qu'elles célèbrent de façon inattendue, audacieuse, intempestive, l'actualité des grands récits, réputés exténués. Habité par les poètes, par les mythes toujours vivaces, Bacon a pu se tenir comme il le disait « au bord du gouffre », parvenant à rendre à la fois le vivant défait par le temps et la mort et la 
résistance de la forme à cette décomposition, d'où l'importance accordée jusqu'au bout aux qualités plastiques: couleurs, dessin, composition (l'usage du triptyque à lui seul est significatif). Son œuvre moderne parce qu'intempestive, intempestive parce que moderne, est la preuve qu'un univers pictural pouvait encore au XXe siècle naitre de la « coagulation» (comme il disait) d'éléments disparates mais prégnants, diachroniques et synchroniques. Les deux moitiés de l'art selon Baudelaire.

\section{BIBLIOGRAPHIE}

\section{Textes cités}

Baldassari, Anne. Bacon/Picasso. Paris : Flammarion/RMN, « La Vie des images », 2005.

Char, René. Fenêtres dormantes et porte sur le toit. Paris : Gallimard, 1979.

Deleuze, Gilles. Logique de la sensation. Paris : Éditions du Seuil, 2002.

Dupin, Jacques. « Notes sur les dernières peintures ». Repères, cahiers d'art contemporain, 39

(1987) : 4-13.

Eliot, T.S. The Complete Poems \& Plays. London: Faber \& Faber, 1969.

Gale, Mattthew \& Stevens Chris. Francis Bacon. Catalogue de la rétrospective Bacon. London: Tate Publishing, 2008.

Genet, Jean. L'Atelier d'Alberto Giacometti. Paris : Gallimard, « L'Arbalète », 1958.

Leiris, Michel. « Ce que m'ont dit les peintures de Francis Bacon ». Au verso des images. Fata Morgana, 1980.

Leiris, Michel. Journal 1922-1989. Paris : Gallimard, 1992.

Mavrakis, Annie. La Figure du Monde, pour une histoire commune de la littérature et de la peinture. Paris : L'Harmattan, 2008.

Peppiatt, Michael. Entretiens avec Francis Bacon, $3^{\mathrm{e}}$ entretien (1989). Trad. Patrice Cotensin. Paris : L'Échoppe, 1998.

Rosenberg, Harold. La Tradition du nouveau. Paris : Éditions de Minuit, 1962.

Sylvester, David. Interviews with Francis Bacon (1975). London: Thames \& Hudson, 2008.

Sylvester, David. Francis Bacon. Paris : Éditions du Centre Pompidou, 1996.

\section{Tableaux cités}

Fig. 1 : Francis Bacon. Study for a Portrait of George Dyer (1967). Huile sur toile. 35,5 x 30,5 cm. Collection privée.

Fig. 2 : Francis Bacon. Three Studies of the Male Back (1970). Huile sur toile. 198 x 147,5 cm. Kunsthaus, Zürich. 
Fig. 3 : Francis Bacon. Seated Figure (1974). Huile sur toile. 198 x 147,5 cm. Collection Gilbert de Botton.

Fig. 4 : Francis Bacon. Three Studies for a Crucifixion (1962). Huile et sable sur toile. Panneau central : 198 x $145 \mathrm{~cm}$. Guggenheim Museum, New York.

Fig. 5 : Francis Bacon. Triptych inspired by Eliot's Sweeney Agonistes (1967). Huile sur toile. 198 x 147,5 cm. Hirshhorn Museum and Sculpture Garden, Smithsonian Institution, Washington.

Fig. 6 : Francis Bacon. Triptych May-June 1973. Huile sur toile. 198 x 147,5 cm. Collection privée, Suisse.

Fig. 7 : Francis Bacon. Triptych inspired by Oresteia of Aeschylus (1981). Huile sur toile. 198 x 147,5 cm. Collection Astrup Fearnley, Oslo, Norvège.

Fig. 8 : Francis Bacon. Study for a Portrait of John Edwards (1986). Huile sur toile. 198 x 147,5 cm. Marlborough Fine Arts.

Fig. 9 : Francis Bacon. Study of the Human Body (1987). Huile sur toile. 198 x 147,5 cm. Collection particulière Paula Traboulsi.

Fig. 10 : Pablo Picasso. Baigneuses ouvrant une cabine de bain (1928). Huile sur toile. 32 x $22 \mathrm{~cm}$. Succession Picasso.

Fig. 11 : Pablo Picasso. Baigneuses jouant au ballon (1928). Huile sur toile. 21,7 x 41,2 cm. Musée Picasso, Paris.

Fig. 12 : Francis Bacon. Three Studies for a Portrait of Isabel Rawsthorne (1967). Huile sur toile. 110,5 x 152,5 cm. Berlin, Alte Nationalgalerie.

Fig. 13 : Francis Bacon. Triptych in memory of George Dyer (1971). Huile sur toile. 198 x 147,5 cm. Fondation Beyerler, Riehen, Bâle

Fig. 14 : Francis Bacon. Painting (Eliot) (1978). Londres, collection privée.

\section{NOTES}

1. Harold Rosenberg, La Tradition du nouveau, Paris : Éditions de Minuit, 1962. On y lit qu'à notre époque «L'échelle des valeurs picturales » cède la place à « la perspective des valeurs finales » et que « l'humanité n'était jamais allée plus loin dans la voie d'une libération de son propre passé » (211).

2. «[...] ce serait une erreur de croire que le peintre travaille sur une surface blanche et vierge. La surface est déjà tout entière investie virtuellement par toutes sortes de clichés avec lesquels il faudra rompre " (Deleuze 19).

3. Comme, pour Leiris, les personnages de Franz Hals par exemple «Ses personnages semblent appartenir à notre temps et non à un passé historique ; ainsi ce sont des personnages totalement présents, - qui sont là et font partie de notre présent ", écrivait-il en 1967 (Leiris 1992, 624, je souligne).

4. L'expression est de René Char, Fenêtres dormantes et porte sur le toit, Paris : Gallimard, 1979.

5. «Non, non, l'œuvre d'art n'est pas destinée aux générations enfants. Elle est offerte à l'innombrable peuple des morts. Qui l'acceptent. Ou qui la refusent. » Jean Genet, L'Atelier d'Alberto Giacometti, Paris : Gallimard, «L'Arbalète », 1958, non paginé.

6. J'ai étudié cette question dans mon livre La Figure du Monde, pour une histoire commune de la littérature et de la peinture, Paris : L'Harmattan, 2008. 
7. Pas même l'École de Londres (Kitaj, Auerbach, Hockney, Freud) à laquelle il est réputé appartenir.

8. On lit aussi «DS: The intention, the Eliot image, that's your private thing. FB: Absolutely. DS: It's not visible in the work. FB: Not at all » (Sylvester 2008, 150).

9. Eliot retrouvait ainsi dans son poème les connotations sinistres, bien éloignées de celle romantiques - d'un Keats, du rossignol antique, auquel est comparée Cassandre dans Agamemnon (voir aussi l'histoire de Philomèle et de Térée chez Ovide).

10. Une autre pièce d'Eliot (The Cocktail Party) accomplit d'ailleurs le scénario de Sweeney Agonistes , une missionnaire manquant de peu de s'y faire dévorer par des cannibales.

11. Voir catalogue de l'exposition de la Tate en 2009, 244.

12. Plusieurs «Baigneuse à la cabine» sont reproduites dans Anne Baldassari, Bacon/Picasso, Paris : Flammarion/RMN, « La Vie des images », 2005, 145 et en particulier la figure 138.

13. "When you're alone in the middle of the night and / you wake in a sweat and a hell of a fright / When you're alone in the middle of the bad and / you wake like someone hit you in the head / You've had a cream of a nightmare dream and / you've got the hoo-ha's coming to you. / Hoo hoo hoo ».

14. Eliot s'est expliqué sur le tarot dans une note à The Waste Land. Au vers 46, il est question d'un « wicked pack of cards » dans lequel Mme Sosostris lit l'avenir (Eliot 62).

\section{RÉSUMÉS}

À propos de la peinture de Francis Bacon et en prenant appui sur des réflexions de Michel Leiris, cet article se propose de revenir sur la question de la modernité conçue non de façon absolue (LA modernité, non figurative, anti-littéraire) mais relative, dégagée d'une vision historiciste orientée de l'histoire de l'art. Cette modernité est intempestive puisque, s'opposant au classique ( cf. La Querelle des Anciens et des Modernes) et non au périmé, elle fait revivre les grandes problématiques esthétiques que l'on pouvait croire obsolètes, telles l'ut pictura poesis. Même si Bacon s'est défendu d'avoir trouvé dans ses poètes de prédilection plus qu'une " atmosphère ", une étude attentive des sujets et des motifs récurrents de ses tableaux dénote une inspiration constamment littéraire. Je m'attache à le montrer dans la seconde partie de cet article en étudiant quelques œuvres qui ne se comprennent qu'en référence à l'Orestie d'Eschyle et à plusieurs textes poétiques ou dramatiques de T.S. Eliot.

This article is about Francis Bacon's painting. It builds on Michel Leiris's remarks and intends to discuss the issue of modernity, not meant as an absolute (the non figurative, anti-literary modernity), but as a relative notion, free of any historicist vision of art history. This modernity is untimely since, in opposition to the classic and not to the out-of-date, it revives the major aesthetic issues, which could be thought obsolete, such as the ut pictura poesis issue. Even if Bacon denied finding in his favorite poets more than an "atmosphere", a careful study of the subject matters and recurring motifs of his paintings indicates a constantly poetical inspiration. That's what I seek to demonstrate in the second part of this article, studying some paintings that can only be understood in reference to Aeschylus' Oresteia and several poetical and dramatic works by T.S. Eliot. 
INDEX

Keywords : ut pictura poesis, modernity, figuration, historicism oeuvrecitee Orestie (L')

Mots-clés : ut pictura poesis, modernité, figuration, historicisme

\section{AUTEURS}

\section{ANNIE MAVRAKIS}

Agrégée de Lettres modernes et docteur en esthétique et sciences de l'art (université Paris I) enseignant en lycée, Annie Mavrakis est spécialiste des relations entre la littérature et l'art. Elle contribue régulièrement à la revue Poétique sur ces questions, ainsi que sur des problèmes théoriques (Recto-Verso, Libres Cahiers pour la Psychanalyse). Auteur de La Figure du Monde, pour une histoire commune de la littérature et de la peinture (L'Harmattan, 2008), elle achève actuellement un ouvrage sur Bacon. 